\title{
Procoagulant Activity of Red Blood Cell-Derived Microvesicles during Red Cell Storage
}

\author{
Akbar Hashemi Tayer ${ }^{a, b}$ Naser Amirizadeh ${ }^{b}$ Minoo Ahmadinejad ${ }^{c}$ Mahin Nikougoftar ${ }^{b}$ \\ Mohammad Reza Deyhim ${ }^{b}$ Sima Zolfaghari ${ }^{b}$
}

a Department of Medicine, Jahrom University of Medical Sciences, Jahrom, Iran;

${ }^{b}$ Blood Transfusion Research Center, High Institute for Research and Education in Transfusion Medicine, Tehran, Iran;

${ }^{c}$ Hematology and Reference Coagulation Lab, Iranian Blood Transfusion Organization, Tehran, Iran

\section{Keywords}

Filtration - Microvesicles · Procoagulant activity ·

Red blood cell storage

\section{Summary}

Background: Red blood cells (RBCs) undergo structural and biochemical alterations during storage which are collectively called RBC storage lesion and cause a decrease in RBC recovery and survival. During storage, erythrocytes release an increasing number of microvesicles (MVs) that have key roles in biological processes. We aimed to investigate the procoagulant activity (PCA) of RBC-derived MVs during storage. Methods: 20 packed RBCs were stored for up to 42 days. Samples were taken at seven different times and evaluated for the presence of RBC-MVs. MVs were separated, and following filtration flow cytometry was used to characterize RBC-MVs based on the expression of glycophorin A (Gly.A) and annexin $\mathrm{V}(\mathrm{AnnV})$ antigens. The coagulant activity of RBC-MVs was tested by clotting time (CT) and PCA assays. Results were compared before and after filtration. Results: Flow cytometry revealed a 17.6-fold increase in RBC-MVs after 6 weeks of storage. Significant correlations were found between AnnV+ MVs and PCA ( $r=0.96$; $p<0.001)$, and CT $(r=-0.77 ; p<0.001)$ which was associated with increased PCA and shortened CT with RBC aging. Filtration of samples efficiently removed MVs $(p<$ $0.001)$ and also reduced in vitro PCA of MVs $(p<0.001)$. Conclusion: RBC-MVs are procoagulant (particularly AnnV+ MVs) Reduction of MVs from RBC concentrates may reduce the risk of transfusion-induced thrombotic complications.

(c) 2018 S. Karger AG, Basel
During the ex vivo storage of red cell concentrates, red blood cells (RBCs) lose their membrane by shedding microvesicles (MVs). MVs, also termed microparticles or ectosomes [1], are small membrane-derived fragments around $0.1-3.0 \mu \mathrm{m}$ in diameter that shed from the cell membrane of various cells during cell activation or apoptosis [2]. Cellular origin of MVs is characterized by cell-specific markers from the parental cells located on their surfaces, such as glycophorin A (Gly.A) and glycoprotein Ib antigens that are used to identify erythrocyte and platelet-derived MVs, respectively $[3,4]$.

MVs consist of different components originating from their parent cells [5]. Phospholipids are the main elements of the cell membrane and also of MV membranes. In resting cells, phospholipids are asymmetrically distributed in the membrane with phosphatidylserine (PS) predominantly in the inner leaflet. This state of the membrane bilayer is regulated by translocating enzymes involving flippase, floppase, and scramblase. Upon cell activation, cytosolic calcium increases and the membrane loses its asymmetric distribution, leading to the exposure of PS on the outer surface and MV formation. The expression of such negatively charged molecules play a key role in the MV shedding process and is also required for the activation of tenase and prothrombinase coagulation complexes, thereby speeding up the coagulation cascade and thrombin generation [6]. Furthermore, MVs are considered to have other biological roles such as involvement in inflammation [7] and vascular function [8]. Blood cell-derived MVs are present at a low level in the circulation of healthy individuals; however, they are elevated in a broad spectrum of pathological conditions such as diabetes, sickle cell anemia, pre-eclampsia, cardiovascular disease, and thrombotic complications [9].

MVs are considered an intrinsic part of all blood products used for transfusion [10]. Several in vitro experiments have

\section{KARGER}

() 2018 S. Karger AG, Basel

Fax +497614520714 
shown that the MV count in RBC concentrates gradually increases over time, suggesting that these MVs might be responsible for the adverse clinical outcome of transfusions $[9,11]$. There is evidence indicating that MVs may be considered a prognostic and diagnostic biomarker of disease [12]. Despite recent improvements in how to identify MVs, there is still a lack of consensus between relevant studies. Therefore, to have a better understanding of MV populations, and also to determine standard definitions around MVs, it seems necessary to do more research on various cell-derived MVs.

A wide variety of methods is used to analyze MVs, including flow cytometry, functional assays, capture-based assays, and proteomic methods. Flow cytometry appears to be the most commonly used method for the assessment of cellular origin and enumerating MVs in physiological and pathological conditions $[3,4]$.

We aimed to characterize RBC-MV populations based on a flow cytometry method and to determine correlations between MVs and procoagulant activity (PCA) and clotting time (CT) assays during RBC storage. In this study, flow cytometric analysis was performed with a precise number of beads to determine MV count, and cell-specific antibodies were used to identify the origin cell of the MVs. We also investigated the impact of filtration to reduce MV count in the samples and to determine what effect this may have on PCA.

\section{Material and Methods}

\section{RBC Preparation and Sampling}

In the current study, packed leuko-depleted RBCs from 20 healthy volunteers who did not have any known coagulation disorder were prepared by the Iranian Blood Transfusion Organization (IBTO) and stored under routine blood banking conditions $\left(4^{\circ} \mathrm{C}\right)$ for 6 weeks. All donors selected had normal screening tests determined by the prothrombin time (PT), activated partial thromboplastin time (APTT), and platelet count. After informed consent, whole blood ( $450 \pm 45 \mathrm{ml}$ ) was obtained through clean phlebotomy of an antecubital vein using 16-gauge needles (BD Vacutainer needles) and collected in to blood bags (Fresenius Kabi, Homburg, Germany), containing $63 \mathrm{ml}$ citrate phosphate dextrose (CPD) as an anticoagulant. The units were then immediately centrifuged at $2,000 \times g$ for $20 \mathrm{~min}$ at $4{ }^{\circ} \mathrm{C}$, to separate plasma; then RBCs were suspended in $100 \mathrm{ml}$ of saline-adenine-glucose-mannitol solution. Leukocytes and platelets were removed by an in-line leukocyte depletion filter. Samples were collected weekly from the RBCs by a sterile sampling device, starting at the day of donation and ending at the expiration date of storage (d0, d7, d14, $\mathrm{d} 21, \mathrm{~d} 28, \mathrm{~d} 35$ and $\mathrm{d} 42$ ).

\section{Isolation and Filtration}

Samples were evaluated for the presence of cell-derived MVs. At first, MVs were isolated from the RBC concentrate with centrifugation. For this purpose, $10 \mathrm{ml}$ of RBCs was mixed with $2 \mathrm{ml}$ of phosphate-buffered saline (pH 7.4) and centrifuged at $2,000 \times g$ for $10 \mathrm{~min}$ at $10^{\circ} \mathrm{C}$. The supernatant was centrifuged again with this protocol to exclude residual RBCs. The centrifuge brake was set to 'off' to prevent mixing RBCs with the supernatant. The top two-thirds of the double-centrifuged plasma was then removed, and half was filtered using a TPP syringe filter $0.22 \mu \mathrm{m}$ (Switzerland) to remove MVs. This was done in order to confirm the action of MVs in the unfiltered aliquots. For the functional assay, pre- and post-filtration samples were stored at $-70{ }^{\circ} \mathrm{C}$ until use.
MV Quantification by Flow Cytometry

Flow cytometric analysis was performed with cy flow space flow cytometry (Partec PAS, Karlsruhe, Germany) using Flomax Software. The instrument has one laser and a standard optical filter setup and photomultiplier voltages are suitable for cellular analysis. Both forward scatter (FSC) and sideward scatter (SSC) have a logarithmic gain.

Using Fluoresbrite ${ }^{\circledR}$ YG Carboxylate Microspheres $1.0 \mu \mathrm{m}$ (Polysciences, Warrington, PA, USA), size events were defined. These tubes contain a precise number of fluorescent beads and allow comparison of measured numbers of MVs between samples.

Phycoerythrin (PE) anti-Gly.A or CD235a (BD Pharmingen, San Diego, CA, USA) and fluorescein isothiocyanate (FITC) anti-human CD42b (BD Pharmingen) were used to tag RBC- and platelet-derived MVs, respectively. Moreover, we used FITC human Annexin V (AnnV) (BD Pharmingen), which tags PS on procoagulant MVs. PE Mouse IgG2b k isotype control (BD Pharmingen) was used as a negative control to differentiate the background noise of the cytometric analysis.

The cellular origin and concentration of MVs were determined immediately pre- and post-filtration of RBC supernatant. Samples $(50 \mu \mathrm{l})$ were labeled with anti-Gly.A ( $5 \mu \mathrm{l})$, anti-CD42b $(5 \mu \mathrm{l})$, and PE-IgG2b isotype control $(5 \mu \mathrm{l})$ for 30 min at $4{ }^{\circ} \mathrm{C}$ in the dark. Also, $50 \mu \mathrm{l}$ of samples were added to test tubes containing $300 \mu \mathrm{l}$ binding buffer $(0.1 \mathrm{~mol} / \mathrm{l} \mathrm{HEPES} \mathrm{/} \mathrm{pH} 7.4,1.4 \mathrm{~mol} / \mathrm{l} \mathrm{NaCl}$, and 25 $\left.\mathrm{mmol} / \mathrm{l} \mathrm{CaCl}_{2}\right)$, and then conjugated AnnV-FITC $(5 \mu \mathrm{l})$ was added. After a 30min incubation at room temperature in the dark, samples were analyzed. Before analysis, $5 \mu \mathrm{l}$ of well-mixed $1.0 \mu \mathrm{m}$ beads that were diluted 1:500 in double-distilled water were added to each sample.

The concentration of RBC-MVs was calculated by comparison to the bead concentration. The number of RBC-MVs was measured in relation to 10,000 beads events. In order to discriminate true events from background noise, MVs were defined by size (less than $0.1 \mu \mathrm{m}$ ), RBC origin (Gly A) and PS exposure $(A n n V+)$ as previously described $[3,4]$. The absolute count of MVs/ $\mu \mathrm{l}$ was calculated as follows:

MVs per $\mu \mathrm{l}=\frac{\text { No. of events in gating containing MVs } \times \text { absolute count of bead per tube }}{\text { No. of events in bead region } \times \text { test volume }}$

\section{Clotting Time Assay}

We also assessed the impact of RBC-MVs on CT. In this study, CT was performed by adding plasma containing RBC-MVs $(100 \mu \mathrm{l})$ to the normal pooled plasma $(100 \mu \mathrm{l})$. The mixture was then kept in a warm water bath for $2 \mathrm{~min}$. Afterward, $100 \mu \mathrm{l}$ of pre-warmed $25 \mathrm{mmol} / \mathrm{l} \mathrm{CaCl}_{2}$ (without any other coagulant factors) was added to the mixture in order to allow clotting to initiate, and then CT was recorded. In the control tube, phosphate-buffered saline was added instead of the MV suspension. Each sample was measured in duplicate.

\section{Determination of PCA}

PCA of MVs was measured using a functional ELISA assay (Zymuphen MPactivity; Hyphen BioMed, Neuville-sur-Oise, France) which is intended to assess PS expression. A microELISA plate is pre-coated with AnnV-streptavidin to capture the MVs from the plasma. Briefly, the calibrator $(100 \mu \mathrm{l})$ and 1:20 diluted pre- and post-filtration samples (containing calcium, factor Xa (FXa) and thrombin inhibitors, $100 \mu \mathrm{l}$ ) were added to the plate wells and then incubated for $1 \mathrm{~h}$ at $37^{\circ} \mathrm{C}$. After 5 washing steps, unbound materials were washed away. Then a mixture containing the clotting factors FXa, FVa, calcium (100 $\mu \mathrm{l})$, and prothrombin $(50 \mu \mathrm{l})$ were added. Procoagulant MVs present in the sample by means of PS bind to AnnV and allow FXa-FVa, to cleave prothrombin into thrombin. After a 10 -min incubation at $37^{\circ} \mathrm{C}$ and addition of specific chromogenic substrate $(50 \mu \mathrm{l})$ and $2 \%$ citric acid as reaction stopping solution $(50 \mu \mathrm{l})$, the amount of generated thrombin that correlates with the number of procoagulant MVs was measured and color was read at $405 \mathrm{~nm}$. Results were calculated according to the calibration curve, and were expressed as nanomolar PS (nM PS) equivalents.

Statistical Analysis

Data are presented as mean + standard deviation (SD). Repeated measure ANOVA, paired t-test, and Pearson correlation were used for data analysis. 

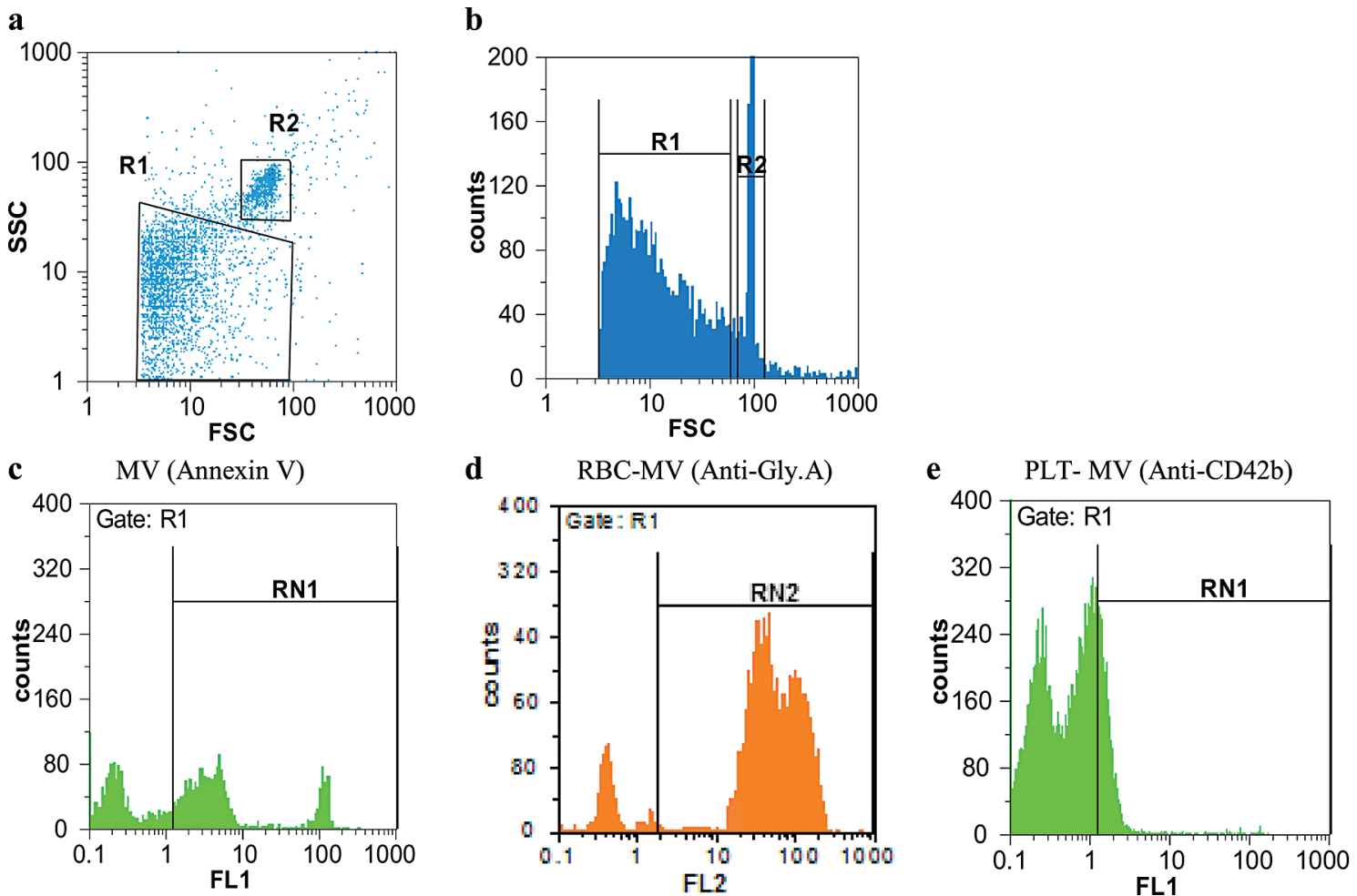

Fig. 1. Flow cytometry plots of RBC-MVs in packed RBCs. FSC and SSC indicates size and granularity, respectively. The MV size gate was set by $1.0 \mu \mathrm{m}$ beads. a Two gating regions are region R1 and R2 that represents MVs and $1.0 \mu \mathrm{m}$ beads, respectively. Based on the FSC and SSC, RBC-MVs are located lower than $1.0 \mu \mathrm{m}$ beads. b A histogram of logarithmic forward scatter versus count, showing the distribution of MVs in comparison to the beads. $\mathbf{c}$ Region RN1 represents region R1 events that were labeled with conjugated FITC-AnnV. d Region RN2 represents region R1 events that were labeled with conjugated PE anti-Gly.A and indicates RBC-derived MVs. e Region RN1 represents region R1 events that were labeled with conjugated FITC anti-CD42b, which indicates platelet-derived MVs. Nonstained events are demonstrated by the left peak in figure $1 \mathrm{c}-\mathrm{e}$.

Table 1. Different microvesicle count/ $\mu l$ of RBC supernatant pre- and postfiltration*

\begin{tabular}{|c|c|c|c|}
\hline & Pre-filtration & Post-filtration & p-value \\
\hline \multicolumn{4}{|c|}{ Total MVs } \\
\hline $\mathrm{d} 0$ & $4,160.1 \pm 1,224.3$ & $404.3 \pm 116.1$ & 0.001 \\
\hline $\mathrm{d} 42$ & $51,087.5 \pm 1,1430.8$ & $6,262.1 \pm 1,591.6$ & 0.001 \\
\hline \multicolumn{4}{|c|}{ Glycophorin A } \\
\hline $\mathrm{d} 0$ & $2,800 \pm 845.3$ & $270.4 \pm 80.4$ & 0.001 \\
\hline $\mathrm{d} 42$ & $49,339 \pm 8,632.1$ & $4,424.5 \pm 1,048.4$ & 0.001 \\
\hline \multicolumn{4}{|c|}{ Annexin V } \\
\hline $\mathrm{d} 0$ & $1,484.5 \pm 355.3$ & $231.9 \pm 68.9$ & 0.001 \\
\hline $\mathrm{d} 42$ & $27,770 \pm 6,097.7$ & $2,281.1 \pm 621.9$ & 0.001 \\
\hline \multicolumn{4}{|l|}{$\mathrm{CD} 42 \mathrm{~b}$} \\
\hline d0 & $1,100 \pm 672.8$ & $113.8 \pm 39.4$ & 0.001 \\
\hline $\mathrm{d} 42$ & $910 \pm 752.4$ & $101.7 \pm 55.3$ & 0.001 \\
\hline
\end{tabular}

*Data are presented as mean \pm standard deviation; $\mathrm{d} 0=$ day 0 ; $\mathrm{d} 42=$ day 42 of storage.
Stata software, version 13 (Stata Corp, College Station, TX, USA) was used for all statistical analysis. Graphs were depicted by Excel and Stata software. P less than 0.05 was considered statistically significant.

\section{Results}

\section{Flow Cytometry}

Flow cytometric analysis of erythrocyte concentrates revealed that all samples tested had MVs. At first, MVs were gated on the basis of their specific FSC and SSC signals (fig. 1a,b). As shown, RBC-MVs were seen as the population slightly smaller and with lower SSC than the $1.0 \mu \mathrm{m}$ beads. Afterward, RBC-MVs were further identified by labeling with fluorescent AnnV (fig. 1c) and Gly A (fig. 1d) markers.

The number of total MVs increased during storage in all cases. Moreover, the difference between the number of MVs on day 42 $(51,085.5 \pm 11,430.8 / \mu \mathrm{l})$ compared to day $0(4,160.1 \pm 1,224.3 / \mu \mathrm{l})$ was statistically significant; MVs increased approximately 12.2 times from day 0 to day 42 ( $p<0.001$; fig. $2 \mathrm{a}$ ). As seen in previous reports, there was a significant variation of the MVs counted between samples that were obtained from different donors [4]. 


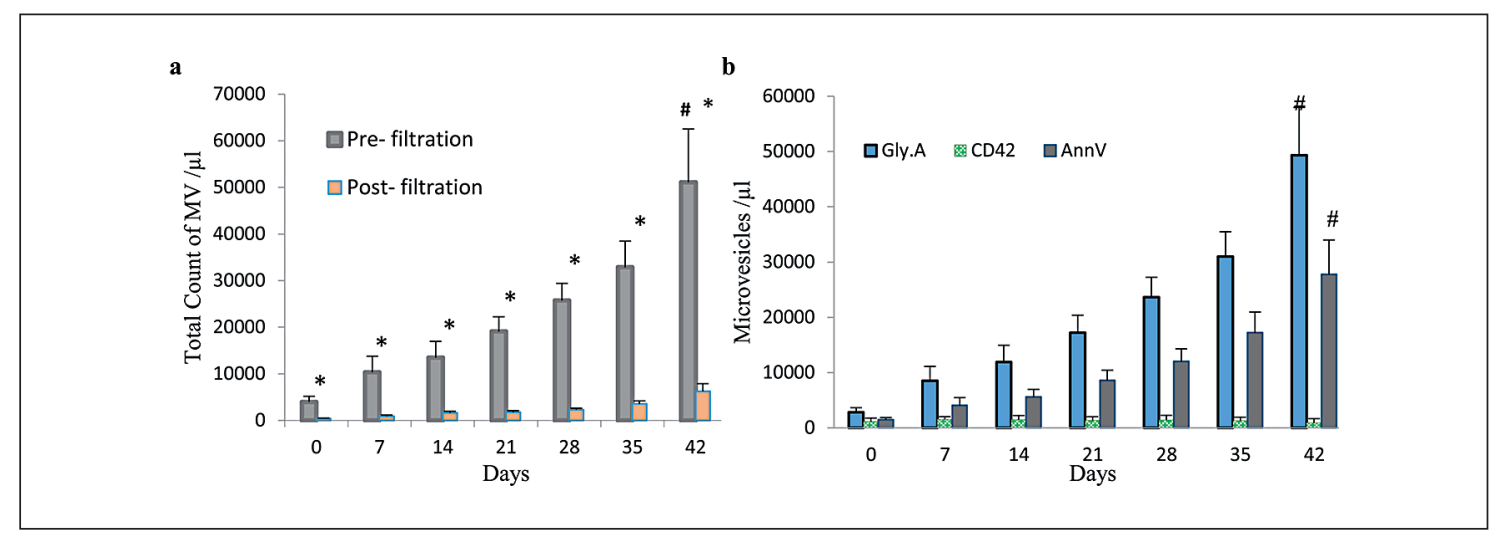

Fig. 2. Quantification of RBC-MVs in supernatant released by RBCs during storage of 20 different packed RBCs. a The number of total MV/ $\mu$ that were counted based on the FSC and SSC. Plot revealed a statistically significant increase of MV number during storage $\left(\mathrm{p}<0.0001\right.$ by ANOVA). ${ }^{*}$ Significant difference $(\mathrm{p}<0.001)$ between pre- and post-filtration MVs, by paired $\mathrm{t}$-test. ${ }^{*}$ Significant change $(\mathrm{p}<0.001)$ over storage period, by ANOVA. b Total count of different MVs according to the expression of Gly.A, CD42b and AnnV that represents RBC, platelet and procoagulant MVs, respectively. Results are mean + SD.

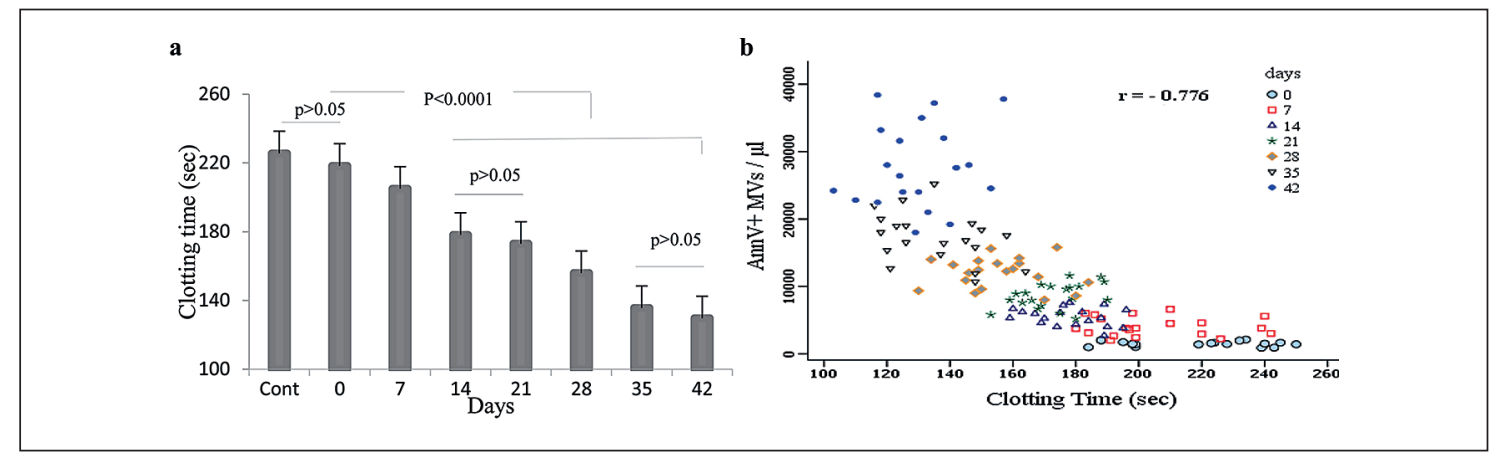

Fig. 3. Assessment of CT on different days of storage and its correlation with the number of AnnV+ MVs in stored RBCs $(n=20)$. a CT changes during storage. The plot showed that CT decreased significantly in all units during storage $(\mathrm{p}<0.001$ by ANOVA). The minimum CT was observed at day 42. b Scatter plot showing the correlation between the number of AnnV+ MVs and CT. There was an inverse relationship between MVs and CT ( $r=-0.776, \mathrm{p}<0.001$ by Pearson's test).

Flow cytometry also showed that the filtration of RBC supernatant reduced approximately $90 \%$ of the number of total MVs (fig. 2a), and of Gly.A-, AnnV-, and CD42b-expressing MVs in samples (table 1).

Measured numbers of Gly.A+ MVs which represent RBC-derived MVs were observed to increase from approximately 2,800 \pm $845.3 / \mu \mathrm{l}$ in fresh samples to approximately $49,339 \pm 8,632.1 / \mu \mathrm{l}$ in older erythrocyte concentrates ( $\mathrm{p}<0.001$; fig. $2 \mathrm{~b}$ ). As expected, the majority of total MVs were of RBC origin and had a positive staining for Gly.A (87.3\%, fig. 1d). The MVs were also positive for AnnV (44\%, fig. 1c) and the platelet marker CD42b (10\%, fig. 1e). The number of MVs positive for platelet marker CD42b was 1,100 $\pm 672.8 / \mu \mathrm{l}$ on day 0 and decreased to $910 \pm 752.4 / \mu \mathrm{l}$ on day 42 which is likely related to the fact that leukocyte-reduced RBCs contain a negligible amount of platelets (fig. 2b).

AnnV is a useful marker to determine the general levels of procoagulant MVs, and the progressive increase in the AnnV+ MVs over the cold storage period was statistically significant (18.7 times; $\mathrm{p}<0.001)$. Although the number of AnnV+ MVs increased during
RBC storage, their mean value was significantly lower than the mean number of total MVs and also RBC-MVs $(\mathrm{p}<0.001)$. Total MVs increased from $1,484.5 \pm 355.3 / \mu \mathrm{l}$ in day 0 and reached to $27,770 \pm 6,097.7 / \mu \mathrm{l}$ on day 42 (fig. $2 \mathrm{~b}$ ). The number of AnnV+ MVs correlated with the number of Gly.A+ MVs ( $r=0.966$; $p<$ 0.001 by Pearson's test).

\section{CT Assay}

Compared with the controls, samples containing MVs had shorter CTs. During storage, procoagulant MVs increased, and there was a significant decrease in the CT. Interestingly, the CT was significantly $(\mathrm{p}<0.001)$ lowered at day 42 compared to day 0 (129.6 \pm 13.8 s vs. $218.3 \pm 22.2$ s) (fig. $3 a)$. As expected, our results showed a significant inverse correlation between $\mathrm{AnnV}+\mathrm{MV}$ counts and CT (fig. 3b) $(r=-0.776 ; p<0.001$ by Pearson's test).

\section{PCA of $M V s$}

The PCA of MVs was significantly increased with longer storage time $(\mathrm{p}<0.001)$, and filtration of supernatants to remove MVs 


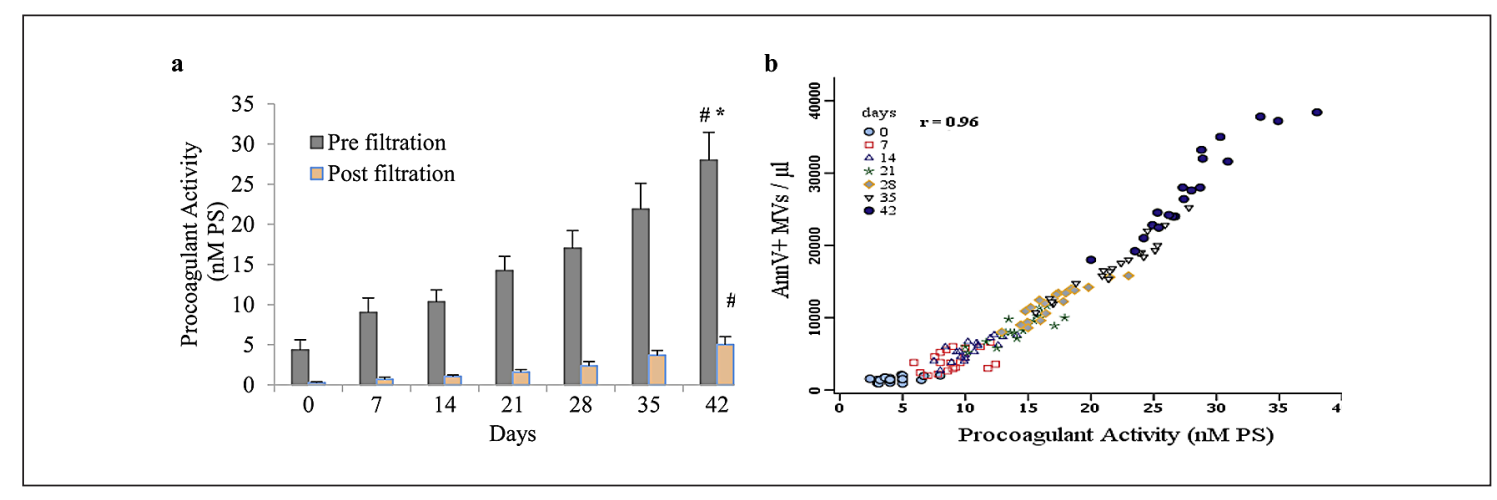

Fig. 4. Assessment of PCA due to PS-expressing MVs and its correlation with the number of AnnV+ MVs in stored RBCs ( $n=20)$. a PCA of pre- and post-filtration samples during storage. The PCA increased significantly over time ( ${ }^{\mathrm{p}}<0.001$; by ANOVA), and there were significant differences between PCA in pre- and post-filtration samples at all times $\left({ }^{*} \mathrm{p}<0.001\right.$; by paired $\mathrm{t}$-test). b Correlation of AnnV+ MVs and PCA measurements. Change in AnnV+ MV number correlated best with the change in PCA ( $r=0.96, p<0.001$ by Pearson's test). Data are presented as mean \pm standard deviation; $\mathrm{d} 0=$ day $0 ; \mathrm{d} 42=$ day 42 of storage.

caused a progressive reduction in PCA in all units. There were significant differences between pre- and post-filtration PCAs at all times tested (fig. $4 \mathrm{a}, \mathrm{p}<0.001$ by t-test) $(4.34 \pm 1.2 \mathrm{nmol} / \mathrm{l}$ vs. 0.25 $\pm 0.1 \mathrm{nmol} / \mathrm{l}$ at $\mathrm{d} 0$ and $27.9 \pm 3.4 \mathrm{nmol} / \mathrm{l}$ vs. $5.01 \pm 0.98 \mathrm{nmol} / \mathrm{l}$ at $\mathrm{d} 42$ ). We found strong and statistically significant associations between PCA and levels of AnnV+ MVs (fig. 4b, r = 0.96; p < 0.001 by Pearson's test) and also RBC-MVs ( $\mathrm{r}=0.95 ; \mathrm{p}<0.001)$.

\section{Discussion}

In this study, we evaluated 20 units of packed RBC concentrates stored in SAGM to identify RBC-MVs and monitor them throughout their storage time.

The clinical importance of MVs has been increasingly identified, emphasizing the need for a better understanding of MVs' biological properties and their roles in disease [9]. Although a wide variety of methods are developed to characterize MVs, generally there is no standardized method to quantify them [13]. In our study, RBC-MVs in stored RBCs were detected using flow cytometry with different antibodies, especially anti-Gly.A and anti-AnnV. These antibodies were selected because they stain specific proteins on the RBC membrane known to be present on RBC-MVs.

Firstly, MVs were quantified on the basis of their size and density, and confirmed using $1.0 \mu \mathrm{m}$ beads [14], and then identified with specific fluorescent antibodies. It seems that MVs located near to the background noise are cell debris. It has been reported that RBC-MVs are smaller than other cell-derived MVs as characterized by electron microscopy [15]. It should be noted that the small size of RBC-MVs is the most common limitation that challenges the sensitivity of flow cytometry methods [16], so when using the method, a limited number of MVs might be calculated.

Our experiments showed a gradual increase in the total count of MVs and also RBC-MVs during storage. Noteworthy, RBC-MV count varied significantly between fresh and 42-day-old samples $(\mathrm{p}<0.001)$. In total, RBC-MVs that expressed Gly.A on their sur- face represented about $87.2 \%$ of the total MVs, and just $10 \%$ of MVs were of platelet origin. It can be suggested that fresh RBC concentrates contain different MVs derived from various cell types and that the gradual increase in the total count of MVs can be contributed only to the release of MVs from RBCs present in the concentrate $[3,4]$. Significant differences in the numbers of RBC-MVs were also identified between donors. The precise reason for this variation remains unknown, but it appears that several factors like $\mathrm{ABO}$ blood group, age, and sex might play a role and should be investigated in future research $[4,17]$. Levels of MVs can also be affected by pre-analytical steps such as sampling, centrifugation, and freezing. Centrifugation is a critical procedure to obtain MVs as it removes cells and debris prior to analysis [18]. For complete removal of RBCs from the supernatant, samples were centrifuged at $2,000 \times g$ for $10 \mathrm{~min}$. In our study, the number of RBC-MVs was associated with duration of storage at $4{ }^{\circ} \mathrm{C}$. Pre-storage leukoreduction also reduces the total number of both RBC-derived MVs and procoagulant particles. In the present study, the exact reasons for the increase in MV counts during storage of RBCs are unknown. However controversial, it has been demonstrated that microvesiculation process could be a way to delay immediate killing of RBCs when they are still viable and functional [19].

Authors suggest that MV changes may be clinically important [20], and further investigations are needed to determine how these changes can be related to the morbidity and mortality associated with blood transfusion. As shown by Koch et al. [21], there is a link between duration of RBC storage and adverse side effects following blood transfusion in cardiac surgery. Based on their finding, transfusion of older erythrocytes in patients undergoing cardiac surgery strongly correlates with the risk of postoperative complications and reduces survival time. However, this issue is still controversial and some of the recently published prospective clinical trials have failed to replicate these findings and limited this link only to particular situations [22]. Increased level of MVs that are associated with coagulation abnormalities have been reported in hemolytic anemias, particularly sickle cell disease and thalassemia [23]. One of the key 
mechanisms contributing to the hypercoagulable state could arise from RBC membrane alteration and subsequent MV formation [24]. Externalization of PS after cell stimulation, cell aging, or in disease states changes the neutral membrane charge into a negative charge offer a docking site for assembly of coagulation enzyme complexes on the procoagulant MV surface [5,6]. Moreover, PS increases the procoagulant ability of tissue factor, which is considered the principal initiator of the coagulation cascade, participating in thrombin generation. Taken together, expression of PS promotes effective hemostasis [25].

For measuring general levels of procoagulant MVs, $\mathrm{AnnV}$ is a useful marker which binds to PS. In our investigation, flow cytometry analysis showed that approximately $44 \%$ of total MVs expressed PS on their surface, and this population increased progressively during storage ( $\mathrm{p}<0.001)$. In a study by Rubin et al. [22], about $96 \%$ of $\mathrm{RBC}-\mathrm{MVs}$ which were isolated from 42-day-stored RBCs were $\mathrm{AnnV}+$. In addition, the amount of AnnV+ events varied greatly between donors, in accordance with previous studies by Dinkla et al. [25], and Connor et al. [26]. They claimed a wide donor variation in the amount of PS in both fresh and stored RBC concentrates, and also showed that different types of MVs exhibit PS at varying levels, while some MVs do not bind to AnnV. Based on PS exposure, RBCMVs could support PCA [27]. We observed that adding supernatant containing RBC-MVs to normal pooled plasma containing calcium strongly reduced CT $(\mathrm{p}<0.001)$. Aged RBC samples created shorter $\mathrm{CT}$ in comparison to fresh samples, and CT significantly correlated with the number of RBC-MVs as storage progressed. This finding is in agreement with the results of Rubin et al. [8], who showed that plasma containing MVs caused a significant decrease in PT. As stated before, MVs have PCA, and it was suggested that transfusion of packed RBCs containing a high number of MVs could induce the risk of thromboembolic complications [28].

In the present study, we also investigated the impact of MVs in filtered samples on the PCA assay. Double-centrifuged samples were filtered using a $0.22 \mu \mathrm{m}$ syringe filter, and the PCA of samples was determined pre- and post-filtration. Filtration removed approximately $90 \%$ of total MVs, and also RBC-MVs; this change in RBC-MVs was associated with a change in AnnV+ MVs. The PCA of MVs was significantly increased during storage $(p<0.001)$, and filtration of samples induced a significant reduction in PCA ( $\mathrm{p}<$ 0.001), indicating that the removal of MVs (especially AnnV+ $\mathrm{MVs}$ ) from samples led to the loss of PCA. Our results are in accordance with a previous study published by Macey et al. [14]. They obtained the same results, showing that filtration of plasma removed $99.8 \%$ of MVs and correlated significantly with reduced thrombin generation. However, these finding were contrary to the report by Aung et al. [29] who concluded that depletion of MVs from packed RBC supernatant using $0.22 \mu \mathrm{m}$ filters reduced the number of AnnV+ MVs but did not change CT and PCA post-filtration, indicating that particles smaller than $0.22 \mu \mathrm{m}$ may increase the PCA. Furthermore, we found statistical associations between PCA and levels of AnnV+ MVs and also RBC-MVs. These findings are supported by previous studies that showed that the expression of PS on MVs is required for thrombin generation [24, 28].

In summary, it can be concluded that storage of RBC concentrates under blood bank conditions is associated with important changes, resulting in significant numbers of MVs originating from RBCs, which have PCA that may affect post-transfusion survival and function of RBCs. This is especially important in the massive blood transfusion setting and also in critically ill patients. In addition, correct quantification and subsequent analysis of RBC-MVs in blood products may be clinically important to provide information about adverse clinical outcomes in transfused recipients. In addition, it can be suggested that removal of thrombogenic MVs from $\mathrm{RBC}$ concentrates may reduce the risk of transfusion-induced blood coagulation and improve the quality of blood products.

\section{Acknowledgments}

This study was supported by 'Iranian High Institute for Research and Education in Transfusion Medicine'. The authors would like to thank all blood donors that have participated in the present study. We appreciate the efforts of Mrs. Golzadeh for technical assistance in flow cytometry experiments, and Mrs. Riahi and her colleagues at the Center for Innovation of IBTO for help in the preparation of RBC units.

\section{Disclosure Statement}

The authors declare no conflicts of interest.

\section{References}

1 Vlassov A, Magdaleno S, Setterquist R, Conrad R: Exosomes: current knowledge of their composition, biological functions, and diagnostic and therapeutic potentials. Biochim Biophys Acta 2012;1820:940-948.

2 Westerman M, Porter JB: Red blood cell-derived microparticles: an overview. Blood Cells Mol Dis 2016;59: 134-139.

3 Grisendi G, Finetti E, Manganaro D, Cordova N, Montagnani G, Spano C, Prapa M, Guarneri V, Otsuru S, Horwitz EM, Mari G, Dominici M: Detection of microparticles from human red blood cells by multiparametric flow cytometry. Blood Transfus 2015;13:274280.
4 Ayers L, Kohler M, Harrison P, Sargent I, Dragovic R, Schaap M, Nieuwland R, Brooks S, Ferry B: Measurement of circulating cell-derived microparticles by flow cytometry: sources of variability within the assay. Thromb Res 2011;127:370-377.

5 Morel O, Jesel L, Freyssinet JM, Toti F: Cellular mechanisms underlying the formation of circulating microparticles. Arterioscler Thromb Vasc Biol 2011;31:1526.

6 Nébor D, Romana M, Santiago R, Vachiery N, Picot J, Broquere C, Chaar V, Doumdo L, Odièvre MH, Benkerrou M, Elion J: Fetal hemoglobin and hydroxycarbamide modulate both plasma concentration and cellular origin of circulating microparticles in sickle cell anemia children. Haematologica 2013;98:862-867.
7 Kent MW, Kelher MR, West FB, Silliman CC: The proinflammatory potential of microparticles in red blood cell units. Transfus Med 2014;24:176-181.

8 Rubin O, Canellini G, Delobel J, Lion N, Tissot JD: Red blood cell microparticles: clinical relevance. Transfus Med Hemother 2012;39:342-347.

9 Van der Pol E, Böing AN, Harrison P, Sturk A, Nieuwland R: Classification, functions, and clinical relevance of extracellular vesicles. Pharmacol Rev 2012;64:676705.

10 Kriebardis AG, Antonelou MH, Georgatzakou HT, Tzounakas VL, Stamoulis KE, Papassideri IS: Microparticles variability in fresh frozen plasma: preparation protocol and storage time effects. Blood Transfus 2016; $14: 228-237$ 
11 Maslanka K, Uhrynowska M, Lopacz P, Wrobel A, Swolenska-Sym G, Guz K, Lachert E, Ostas A, Brojer E: Analysis of leukocyte antibodies, cytokines, lysophospholipids and cell microparticles in blood components implicated in post-transfusion reactions with dyspnoea. Vox Sang 2015;108:27-36.

12 Tesselaar ME, Romijn FP, Van Der Linden IK, Prins FA, Bertina RM, Osanto S: Microparticle-associated tissue factor activity: a link between cancer and thrombosis. J Thromb Haemost 2007;5:520-527.

13 Lacroix R, Dignat-Georges FD: Microparticles as a circulating source of procoagulant and fibrinolytic activities in the circulation. Thromb Res 2012;129:27-29.

14 Macey MG, Enniks N, Bevan S: Flow cytometric analysis of microparticle phenotype and their role in thrombin generation. Cytometry B Clin Cytom 2011;80:5763.

15 Livaja Koshiar R, Somajo S, Norström E, Dahlbäck B Erythrocyte-derived microparticles supporting activated protein $\mathrm{C}$-mediated regulation of blood coagulation. PLoS One 2014;9(8): e104200.

16 Nielsen MH, Beck-Nielsen H, Andersen MN, Handberg A: A flow cytometric method for characterization of circulating cell-derived microparticles in plasma. J Extracell Vesicles 2014;3:doi: 10.3402/jev.v3.20795.

17 Rubin O, Crettaz D, Tissot JD, Lion N: Microparticles in stored red blood cells: submicron clotting bombs? Blood Transfus 2010;8:31-38.
Chandler WL: Microparticle counts in platelet-rich and platelet-free plasma, effect of centrifugation and sample-processing protocols. Blood Coagul Fibrinolysis 2013;24:125-132.

19 Willekens FL, Werre JM, Groenen-Dopp YA, Roerdinkholder-Stoelwinder B, Pauw BD, Bosman GJC: Erythrocyte vesiculation: a self-protective mechanism? Br J Haematol 2008; 141:549-556.

20 Burnouf T, Chou ML, Goubran H, Cognasse F, Garraud $\mathrm{O}$, Seghatchian J: An overview of the role of microparticles/microvesicles in blood components: are they clinically beneficial or harmful? Transfus Apher Sci 2015;53:137-145.

21 Koch CG, Li L, Sessler DI, Figueroa P, Hoeltge GA, Mihaljevic T, Blackstone EH: Duration of red-cell storage and complications after cardiac surgery. $\mathrm{N}$ Engl J Med 2008;358:1229-1239.

22 Rubin O, Delobel J, Prudent M, Lion N, Kohl K, Tucker E: Red blood cell-derived microparticles isolated from blood units' initiate and propagate thrombin generation. Transfusion 2013;53:1744-1754

23 Van Beers EJ, Schaap MC, Berckmans RJ, NieuwlandR, Sturk A, van Doormaal FF, Meijers JC, Biemond BJ: Circulating erythrocyte derived microparticles are associated with coagulation activation in sickle cell disease. Haematologica 2009;94:1513-1519.
24 Zayed RA, El-Ghamrawi M, Alwakeel HA, Esh N: Impact of circulating erythrocyte-derived microparticles on coagulation activation in sickle cell disease. Comp Clin Pathol 2015;24:1123-1128.

25 Dinkla S, Peppelman M, Van Der Raadt J, Atsma F, Novotný VM, Van Kraaij MG, Joosten I, Bosman GJ: Phosphatidylserine exposure on stored red blood cells as a parameter for donor-dependent variation in product quality. Blood Transfus 2014;12:204-249.

6 Connor DE, Exner T, Ma DDF, Joseph JE: The majority of circulating platelet-derived microparticles fail to bind annexin V, lack phospholipid dependent procoagulant activity and demonstrate greater expression of glycoprotein Ib. Thromb Haemost 2010;103:10441052

27 Lacroix R, Judicone C, Poncelet P, Robert S, Arnaud L, Sampol J, Dignat-George F: Impact of pre-analytical parameters on the measurement of circulating microparticles: towards standardization of protocol. Thromb Haemost 2012;10:437-446.

28 van der Meijden PEJ, van Schilfgaarde M, Van Oerle R, Renné T, Ten Cate H, Spronk HM: Platelet- and erythrocyte-derived microparticles trigger thrombin generation via factor XIIa. J Thromb Haemost 2012;10: 1355-1362.

29 Aung HH, Tung JP, Dean MM, Flower RL, Pecheniuk NM: Procoagulant role of microparticles in routine storage of packed red blood cells: potential risk for prothrombotic post-transfusion complications. $\mathrm{Pa}$ thology 2017;49:62-69. 